\title{
Coding for Two-User Energy Harvesting Interference Channel
}

\author{
Mehdi Dabirnia $^{\circledR}$, Member, IEEE, and Tolga M. Duman ${ }^{\circledR}$, Fellow, IEEE
}

\begin{abstract}
A two-user interference channel with energy harvesting transmitters, each equipped with a finite battery, is considered. Achievable rate regions (ARRs) considering independent and identically distributed Shannon strategies at both users and ignoring the memory in the battery state are obtained for both single-user decoding and joint decoding at the receivers. Explicit and implementable codes based on concatenation of a nonlinear trellis code (NLTC) with an outer low-density paritycheck code are designed, and it is demonstrated that rate pairs close to the boundary of ARR can be obtained with this approach. Furthermore, an improved alternative decoding scheme which exploits the memory in the battery state is developed, and it is shown to be highly superior to the simple decoding approach via numerical examples. Superiority of the newly developed practical channel coding solutions over the previously known alternative approaches are illustrated via extensive set of examples as well.
\end{abstract}

Index Terms-Energy harvesting communication systems, nonlinear trellis codes, LDPC code design, channels with memory.

\section{INTRODUCTION}

$\mathbf{M}$ ANY wireless communication and networking applications such as Internet of Energy Neutral Things (IoENT), radio frequency identification (RFID) systems, wireless sensor networks (WSNs), and wireless body networks can benefit from energy harvesting (EH). In an energy harvesting communication system, the transmitter derives its energy from an external source, and it either utilizes the harvested energy for immediate information transmission or stores it in battery for subsequent transmissions. While energy harvesting has significant potential benefits, it imposes new constraints on the design and implementation of communication systems in a variety of forms. For example, stochastic nature of energy harvests in ambient energy harvesting communication systems imposes different transmission constraints compared

Manuscript received June 17, 2019; revised November 27, 2019; accepted January 17, 2020. Date of publication January 28, 2020; date of current version August 19, 2020. The work of Tolga M. Duman was supported in part by the Turkish Scientific and Technological Research Council of Turkey (TUBITAK) BIDEB Program. This article was presented in part at the IEEE International Symposium on Information Theory, Aachen, Germany, June 2017. Part of this article is based on the Ph.D. dissertation of M. Dabirnia completed at Bilkent University. The associate editor coordinating the review of this article and approving it for publication was D. Gunduz. (Corresponding author: Mehdi Dabirnia.)

Mehdi Dabirnia is with the Department of Information and Communications Technologies, Universitat Pompeu Fabra, 08018 Barcelona, Spain (e-mail: mehdi.dabirnia@upf.edu).

Tolga M. Duman is with the Department of Electrical and Electronics Engineering, Bilkent University, 06800 Ankara, Turkey (e-mail: duman@ee.bilkent.edu.tr).

Digital Object Identifier 10.1109/TGCN.2020.2969786 to the classical communication systems where the energy is available upfront. In addition to the randomness in the availability of energy for transmission, the specific realizations of energy arrivals and energy state of the transmitter are also unknown to the receiver. Therefore, there can be errors induced by lack of energy in addition to those introduced by the channel. As a result, transmission schemes and coding solutions developed for standard communication systems are not suitable for EH systems, and it is imperative to design novel coding algorithms to achieve reliable transmission in practice.

Energy harvesting communication systems have recently received significant attention, mostly from information and communication theoretic points of view for both single-user and multi-user set-ups. The additive white Gaussian noise (AWGN) channel capacity is studied for single-user EH communications with infinite battery and zero battery in [1], [2], respectively. Finite size battery case is studied in [3]-[5], and approximations along with bounds on the capacity are obtained. Noiseless binary energy harvesting channel with unit-sized battery is studied in [6], and computable upper and lower bounds on its capacity are obtained. Achievable rates for energy harvesting intersymbol interference channel with finite-alphabet channel inputs are given in [7]. Optimal transmission policies for point-to-point communication under different EH constraints are investigated in [8]-[10]. A learning theoretic approach for obtaining optimal transmission strategies is considered in [11]. A connection between the information and communication theoretic formulations of the single-user EH communications is provided in [4], and it is shown that information theoretic capacity of an energy harvesting channel is within a constant gap of its long-term average throughput.

Capacity of EH Gaussian multiple access channel (MAC) with finite batteries is studied in [12], and it is shown that the sum-capacity approaches that of a standard additive white Gaussian noise MAC as the number of users in the MAC is increased. Optimal transmission policies for EH multiple access channels are investigated in [13], [14]. Two-user Gaussian interference channel with EH transmitters is considered in [15], and power allocation policies maximizing the sum throughput are found. Symmetric two-user Gaussian interference channel with EH transmitters and energy cooperation is studied in [16], and an achievable average rate region is characterized employing Han-Kobayashi (HK) scheme. Specifically, it is shown that allowing the energy cooperation enlarges the achievable average rate region. 
In the current literature, the focus of energy harvesting communications research has been on finding the transmission limits for different channel models under specific constraints on the EH process (information theoretic perspective), and finding optimal policies to maximize the system throughput (communication theoretic perspective). The studies considering practical schemes with finite alphabet inputs and explicit code designs for EH communications are very limited, see [17]-[19] for the single-user case. With this motivation, complementary to the current literature, in this paper, we focus on explicit code design for two-user EH interference channel. Specifically, we consider two EH transmitters, each equipped with a finite battery, sending data over a noisy interference channel using independent and identically distributed (i.i.d.) on-off inputs. We assume that energy arrivals are i.i.d., and that causal energy arrival information is available only at the transmitter. A preliminary version of this study which focuses on the single-user case was presented in [17]. We consider the use of i.i.d. Shannon strategies at both users while ignoring the memory in the battery state, and obtain achievable rates based on the HK scheme. We employ a simpler version which utilizes only private or only public messages for both users with single-user decoding or joint decoding at the receivers. We observe that a larger rate region can be obtained by using non-uniform input distributions for both users, and motivated by this observation, we propose an encoding scheme based on concatenation of an inner nonlinear trellis code (NLTC) with an outer low density parity check (LDPC) code for EH communications over ICs. The inner nonlinear code is used to provide the optimal ones' density while the outer linear code offers strong error correction capabilities. We note that, other approaches, e.g., based on "probabilistic shaping" [20] or "constrained coding" [21] can also be employed. As a simple decoding approach, we ignore the memory in the battery state, i.e., we model the received signal to be through a channel with i.i.d. states, and develop a decoder based on average statistics of the channel state. Due to its simplicity, this decoding scheme is utilized for code design purposes as well, i.e., for finding the optimal LDPC code ensembles for use over the interference channel with EH transmitters.

Another main contribution of this paper is development of an improved decoding scheme for the system model under consideration. That is, in order to take advantage of the memory in the battery state at the decoder side, we build an extended trellis representing both the NLTCs and battery states, and perform a Bahl, Cocke, Jelinek and Raviv (BCJR) algorithm based decoding. Through several numerical examples, we demonstrate that this more sophisticated approach is highly superior to the simple one which ignores the memory in the system.

The paper is organized as follows. In Section II, we introduce the system model for an energy harvesting communication system operating over an interference channel. We provide achievable rate regions with i.i.d. Shannon strategies for different channel models in Section III. Newly proposed explicit coding schemes are described in Section IV along with the specific code design procedure and the improved decoding algorithm. We provide several design examples and decoding

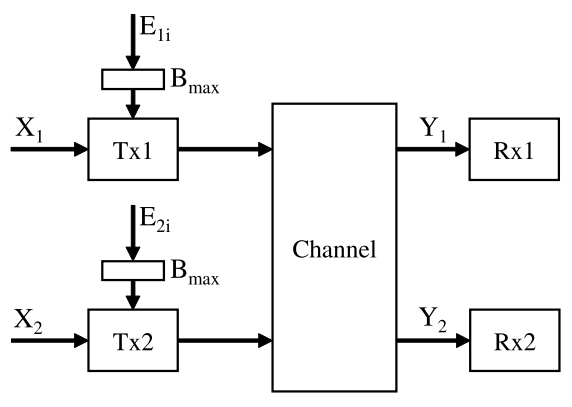

Fig. 1. A two-user EH communication system.

performance results in Section V, and finally, we conclude the paper in Section VI.

\section{SySTEM MOdEL}

We consider a two-user interference channel with energy harvesting transmitters and independent energy arrivals at each user as shown in Fig. 1. We adopt the energy harvesting model introduced in [6], wherein at time instant $i$ an energy packet arrives at the $j$ th transmitter with probability $q_{j}$ independent of the energy arrivals at the other user and other time instances. That is, the EH is governed by two i.i.d. binary energy arrival processes denoted by $E_{j i} \sim$ $\operatorname{Bernoulli}\left(q_{j}\right), j \in\{1,2\}$. The channel inputs $X_{j i}$ are also binary with either zero or one unit energy cost. Each energy harvesting transmitter is equipped with a finite-sized battery for which the battery size is a multiple of the fixed unit of energy harvests (denoted by $B_{\max }$ ). Note that, even though we assume an i.i.d. energy arrival process, the specific coding scheme proposed in Section IV can be extended to other energy arrival models such as those incorporating Markov arrivals as well.

By observing the battery state $S_{j i}$, for each channel use, the transmitter $j$ first transmits a symbol $X_{j i}$, and then it harvests energy $E_{j i}$ and stores it in its battery if there is space. If the battery is empty, regardless of what the input bit is, $X_{j i}=0$ is transmitted. The battery state evolves as

$$
S_{j i+1}=\min \left\{S_{j i}-X_{j i}+E_{j i}, B_{\max }\right\},
$$

which is causally known at the transmitter side only.

For a single-user state dependent channel with causal state information at the transmitter only, if the channel states are i.i.d. and independent of the input, Shannon strategies [22] achieve the capacity. However, for the EH channel, the battery state depends on the channel input and previous state, hence it has memory. Authors in [3] show that, although i.i.d. Shannon strategies are not optimal, they can be used to achieve good transmission rates for a single-user EH channel. With this motivation, in the following, we study achievable rates for both single-user case and the interference channel set-up with EH transmitters using i.i.d. Shannon strategies.

\section{Achievable Rates}

A simple achievable scheme for a single-user EH communication system using i.i.d. Shannon strategies has been 
previously considered in [3], [6] where the authors utilize i.i.d. strategies that depend only on the current battery state. This approach is justified by the Shannon-MacMillan-Brieman theorem which also applies to our set-up. Another simplification considered in [6] is that the decoder ignores the memory in battery states and treats the channel as if it is memoryless which results in a scheme called naive i.i.d. Shannon strategies (NIID). In the following, we first study the achievable rates based on the NIID scheme for a single-user EH channel, and then extend it to the two-user EH interference channel (EHIC) model.

\section{A. Achievable Rates for Single-User EH Channel}

We consider an encoding scheme with i.i.d. Shannon strategies. Specifically, we only consider two possible strategies, $U=\left(X_{0}, X_{1}, \ldots, X_{B_{\max }}\right) \in\{(0,0, \ldots, 0),(0,1, \ldots, 1)\}$ where $X_{i}$ is the channel input when the battery state is $S=i$, namely, these strategies are based on partial state information regarding empty vs. non-empty battery. Let us denote $U=(0,0, \ldots, 0)$ with 0 and $U=(0,1, \ldots, 1)$ with 1 , and utilize an i.i.d. $U_{i}$ sequence with $P\left[U_{i}=1\right]=p$ which is equivalent to using i.i.d. on-off inputs with ones' density $p$. Since the energy arrivals are i.i.d. (with arrival probability $q$ ), the battery state process can be modelled as a Markov chain shown in Fig. 2, which is irreducible and aperiodic. Therefore, it has a stationary probability distribution denoted by $\left\{\pi_{i}\right\}_{i=0}^{B_{\max }}$ where $\pi_{i}=P(S=i)$ is the steady state probability of the Markov chain being in state $i$, given by

$$
\begin{aligned}
\pi_{0} & =\frac{(1-q) p}{(1-q) p+q \sum_{i=0}^{B_{\max }-1}\left(\frac{q(1-p)}{p(1-q)}\right)^{i}} \\
\pi_{i} & =\frac{1}{1-q}\left(\frac{q(1-p)}{p(1-q)}\right)^{i} \pi_{0}, \quad i=1, \ldots, B_{\max } .
\end{aligned}
$$

As a simple approach, the decoder ignores the memory in the battery states, and assumes that the equivalent channel evolves as a memoryless one. It performs decoding using the average statistics of the channel state given in (2)-(3) rather than its $n$-letter evolution. This is equivalent to decoding over a channel obtained by combination of a memoryless Z-channel of crossover probability $\pi_{0}$ with the original one.

As an illustration, we consider a single-user energy harvesting transmitter operating over a binary symmetric channel (BSC) with crossover probability $\epsilon$. The achievable rate for this model is given as

$$
\begin{aligned}
R & =\max _{p \in[0,1]} H(Y)-H(Y \mid X) \\
& =\max _{p \in[0,1]} H_{b}\left(p\left(\bar{\pi}_{0} * \epsilon\right)+\bar{p} \epsilon\right)-p H_{b}\left(\bar{\pi}_{0} * \epsilon\right)-\bar{p} H_{b}(\epsilon)
\end{aligned}
$$

where $\bar{x}=1-x, H_{b}(p)=-p \log _{2}(p)-\bar{p} \log _{2}(\bar{p})$, $P(X=1)=p$, and $x * y=x(1-y)+(1-x) y$.

The optimal ones' density $p$ in (4) for a BSC $(0.1)$ is depicted in Fig. 3. Clearly, the optimal ones' density in most cases is not uniform, which suggests that using linear block codes, which induce a uniform input distribution of "0"s and " 1 "s,
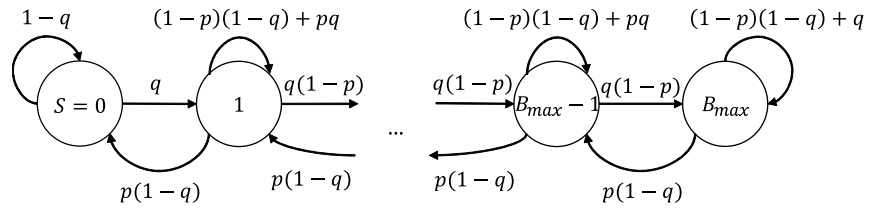

Fig. 2. Markov chain model of the battery state with battery capacity $B_{\max }$.

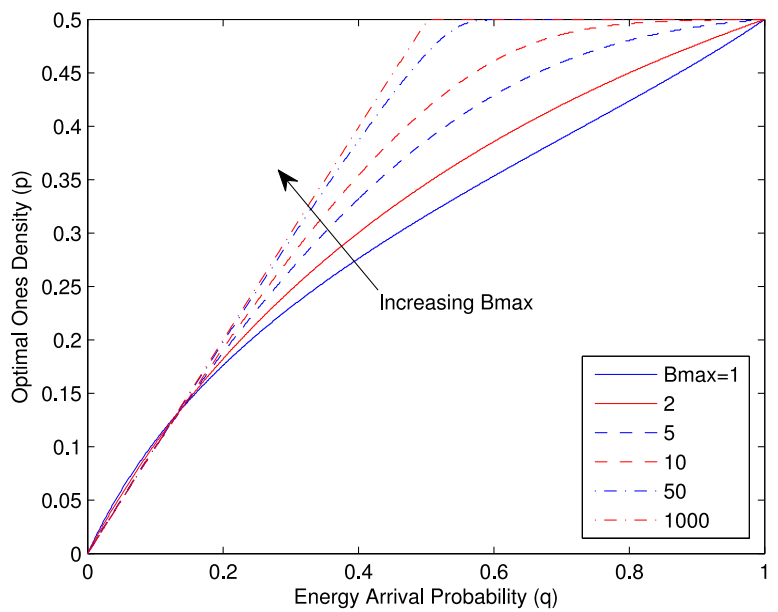

Fig. 3. Optimal ones' density for the NIID scheme for energy harvesting $\operatorname{BSC}(0.1)$

by themselves are not optimal. Namely, one can obtain higher rates by using nonlinear codes.

For the above example, when the battery capacity increases, the optimal ones' density approaches the energy arrival probability for the interval $0<q<0.5$, and it becomes uniform for $0.5 \leq q \leq 1$. Another interesting observation is that when the battery capacity is small and energy arrival probability is also very small, e.g., $q=0.12$ or lower, the optimal ones' density is larger than the energy arrival probability.

\section{B. Achievable Rate Region for EH Interference Channel}

We now consider the encoding scheme adopting i.i.d. on-off signaling at both users with ones' density $P\left(X_{j i}=1\right)=p_{j}$, and obtain achievable rates. Similar to the single-user case, the battery states of each user can be modelled as a Markov chain for which the steady state probabilities are easily derived. Also, we assume that both decoders ignore the memory in the battery state and perform decoding assuming an equivalent memoryless channel as in the single-user case. Therefore, we can utilize a suitable HK scheme on this simplified model to obtain an achievable rate region for the original two-user EHIC. Although a general HK type encoding using both private and public messages for each user can be utilized, here we consider a simpler version by utilizing only private or only public messages for each user.

For illustration, we consider an instance of the discrete memoryless interference channel with energy harvesting transmitters and calculate the achievable rate region with different 


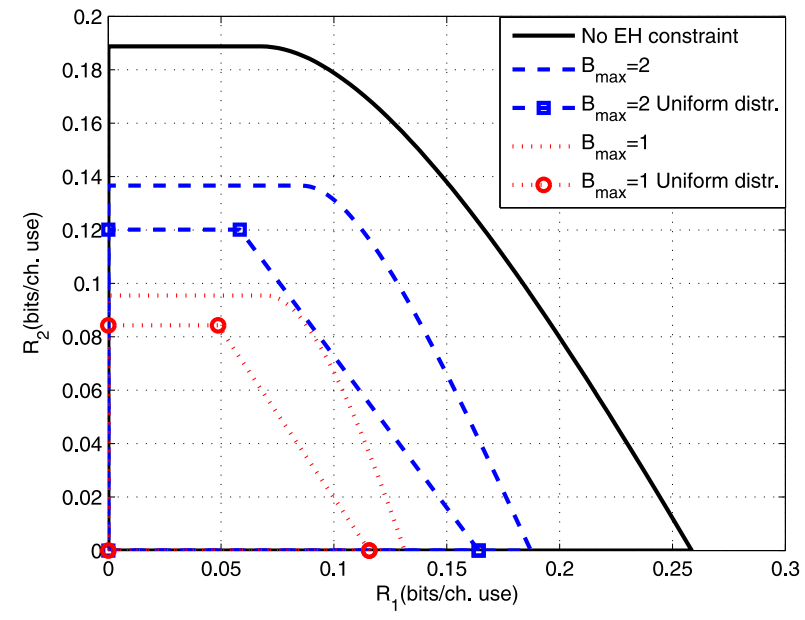

Fig. 4. The ARR for two-user EHIC (5) with two different battery capacities and $q=0.5$.

parameters. For this specific example the input-output relationship is given by

$$
\left\{\begin{array}{l}
Y_{1}=\left(X_{1} \otimes X_{2}\right) \oplus Z_{1} \\
Y_{2}=X_{2} \oplus Z_{2}
\end{array}\right.
$$

where $\oplus$ and $\otimes$ represent the XOR and the OR operations, respectively, with $Z_{1}$ and $Z_{2}$ being the noise samples at receiver 1 and 2 drawn from a Bernoulli distribution with parameters $\epsilon_{1}=0.21$ and $\epsilon_{2}=0.25$, respectively. Achievable rates using single-user decoding at both users for a specific input distribution (for fixed $p_{1}$ and $p_{2}$ ) is given by

$$
\begin{aligned}
R_{1}= & H_{b}\left(p_{1}\left(\bar{\pi}_{10} \bar{\epsilon}_{1}+\pi_{10}\left(p_{2} \bar{\pi}_{20} * \epsilon_{1}\right)\right)+\bar{p}_{1}\left(p_{2} \bar{\pi}_{20} * \epsilon_{1}\right)\right) \\
& -p_{1} H_{b}\left(\bar{\pi}_{10} \bar{\epsilon}_{1}+\pi_{10}\left(p_{2} \bar{\pi}_{20} * \epsilon_{1}\right)\right)-\bar{p}_{1} H_{b}\left(p_{2} \bar{\pi}_{20} * \epsilon_{1}\right), \\
R_{2}= & H_{b}\left(p_{2}\left(\bar{\pi}_{20} * \epsilon_{2}\right)+\bar{p}_{2} \epsilon_{2}\right)-p_{2} H_{b}\left(\bar{\pi}_{20} * \epsilon_{2}\right)-\bar{p}_{2} H_{b}\left(\epsilon_{2}\right),
\end{aligned}
$$

where $\bar{x}=1-x$ and $x * y=x(1-y)+(1-x) y$. The ARR corresponding to this scheme is obtained by taking the convex hull of all of the subregions corresponding to different $p_{1}, p_{2}$ values with $0 \leq p_{1}, p_{2} \leq 1$.

The ARRs for single and two unit battery capacities and energy arrival probability $q=0.5$ are shown in Fig. 4 . The results demonstrate that even by using a unit battery at the transmitters we can still achieve a significantly large portion of the ARR obtained with no energy harvesting constraints. The ARRs corresponding to uniform and identically distributed inputs are also shown on the same figure, clearly demonstrating that significant improvements can be obtained by utilizing codebooks with nonuniform input distributions for the present setup.

As another example, we consider a two-user EH Gaussian interference channel with channel coefficients (from the $j$ th transmitter to the $i$ th receiver) $h_{j i}$, and noise variance $\sigma_{i}^{2}$ for $i, j \in\{1,2\}$. The signal-to-noise ratio (SNR) and interference-to-noise ratio (INR) for this channel are defined as $\mathrm{SNR}_{i}=\frac{h_{i i}^{2}}{2 \sigma_{i}^{2}}$ and $\mathrm{INR}_{i}=\frac{h_{j i}^{2}}{2 \sigma_{i}^{2}}$, respectively. The ARR for this model using i.i.d. on-off signaling with $P\left(X_{i}=1\right)=p_{i}$, $i=1,2$, at the inputs can be obtained as

$$
R_{i}=I\left(Y_{i} ; X_{i}\right)=h\left(Y_{i}\right)-h\left(Y_{i} \mid X_{i}\right),
$$

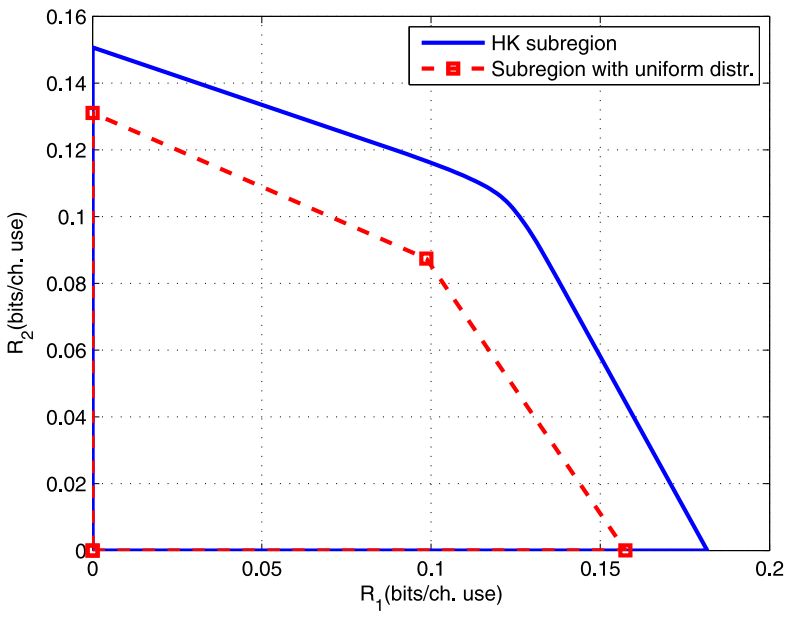

Fig. 5. The ARR for Gaussian channel case with $q=0.5, S N R_{1}=1$, $S N R_{2}=0, I N R_{1}=2$ and $I N R_{2}=1$.

where the distributions of $Y_{i}$ and $Y_{i} \mid X_{i}$ are given in (6) and (7) respectively, shown at the bottom of the next page, and $\mathcal{N}\left(\mu, \sigma^{2}\right)$ denotes the Gaussian PDF with mean $\mu$ and variance $\sigma^{2}$. Fig. 5 depicts the ARR for the special case of $q=0.5, S N R_{1}=1$, $S N R_{2}=0, I N R_{1}=2$ and $I N R_{2}=1$. We observe that, as in the case of single-user EH communications, a significantly large ARR compared to that obtained by uniform inputs can be achieved by utilizing a nonuniform input distribution, motivating development of nonlinear coding solutions.

\section{Practical Coding Scheme for EH COMMUNICATIONS}

As described in the previous section for both single-user and two-user EH interference channels, in order to achieve higher rates by using the NIID scheme, specific nonuniform input distributions need to be employed. To generate the required nonuniform input distribution and also to obtain good error correction performance, we now propose concatenation of an outer linear block code such as an LDPC code with an inner nonlinear trellis code as a practical approach.

Fig. 6 depicts the block diagram of the proposed coding scheme. The transmitter side consists of concatenation of an outer LDPC encoder with an inner nonlinear trellis encoder with a battery state conditioner, which is then connected to the channel. Battery state conditioner applies the battery outage effect, i.e., it simply transmits the symbol 0 when the battery is empty. The goal of the inner NLTC is to induce the desired nonuniform distribution at the channel input while providing error protection against channel noise. The outer LDPC code is intended to provide error correction capabilities.

At the receiver side, as a simple approach, we first ignore the memory in the channel state and consider a channel with i.i.d. states with stationary probabilities given in (2). The decoder assumes that a memoryless $\mathrm{Z}$ channel with crossover $1 \rightarrow 0$ probability $\pi_{0}$ is connected to the memoryless noisy channel (which is either a BSC or an AWGN channel in our setting). A BCJR algorithm based decoder is adopted for the two step memoryless channel computing the log-likelihood-ratios (LLRs) of the encoded bits to be fed to the LDPC decoder. 


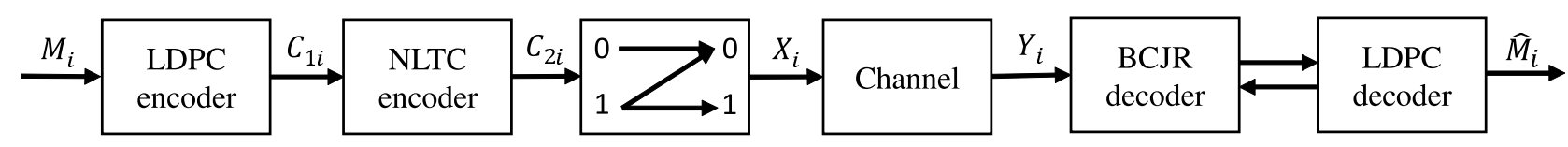

Fig. 6. Block diagram of the proposed coding scheme.

To improve the decoding performance, the soft outputs of the LDPC decoder are fed back to the BCJR decoder in an iterative fashion as also illustrated in Fig. 6.

While the decoding scheme described above is used for code design purposes, we also introduce another (improved) iterative decoding solution in Section IV-C which exploits the memory in the battery state.

\section{A. Inner NLTC Design}

To design the inner NLTC, we utilize the algorithm developed in [23], which maximizes the minimum distance of the code while keeping a desired ones' density. As it is stated in [24], having a larger minimum distance helps with the stability of the iterative decoding process. Also, it is a valid criterion for designing trellis-base codes for point-topoint $(\mathrm{P} 2 \mathrm{P})$ scenarios. The output of the design algorithm is a lookup table which assigns specific labels to the branches of the trellis in such a way that ensures the specific distribution of ones and zeros, and provides a large minimum distance.

\section{B. Outer LDPC Code Design}

Off-the-shelf LDPC codes that are optimized for P2P communication scenarios (e.g., for AWGN channels) are not optimal for the case of energy harvesting communication systems, that is, one needs to optimize the degree distribution of the LDPC ensemble (for each specific energy harvesting scenario). To accomplish this, we fix the inner NLTC (which is specifically designed to provide the optimal ones' density for the particular system parameters, e.g., battery size, energy arrival probability), and perform optimization of the LDPC degree distribution. LDPC code optimization can be performed based on different objectives such as decoding SNR threshold minimization or rate maximization. In this paper, we employ the latter approach. Among different algorithms and techniques available, we utilize the EXIT analysis [25] to measure the iterative decoding threshold of the concatenated coding scheme, and employ a random perturbation algorithm to generate new instances of the LDPC degree distribution.

We assume symmetry of the PDF of exchanged LLRs between the component decoders, and perform the EXIT analysis by using Monte Carlo simulations without any assumption on their Gaussianity [24]. We follow the approach taken in [26] and compute the extrinsic mutual information using

$$
I(L ; X) \approx 1-\frac{1}{N} \sum_{n=1}^{N} \log _{2}\left(1+e^{-L_{n}}\right)
$$

with $L_{n}$ denoting the LLR corresponding to the $n$th coded bit. We utilize i.i.d. channel adaptors introduced in [27] with common randomness at the transmitter and the receiver sides for each message to be able to use the all-zero codewords. We use Monte Carlo simulations for sufficiently long block lengths (taken as $10^{6}$ in this study) for the iterative decoder, and check for convergence by tracking the evolution of the mutual information at the output of the component decoders. We use perturbing vectors to generate new instances of the degree distributions with the goal of maximizing the rate of the code. We terminate the optimization procedure if no improvement can be obtained after a predetermined number of iterations. Further details about the LDPC code design by using the adopted random perturbation technique can be found in [24], [28].

\section{An Improved Iterative Decoding Algorithm}

As a second decoding approach, we propose building an extended trellis by including the battery state at the beginning of each trellis section along with the NLTC states which expands the size of the trellis by a factor of $B_{\max }+1$. That is, for each NLTC state, there are $B_{\max }+1$ corresponding states in the extended trellis, namely, one for each battery state in $\left\{0,1, \ldots, B_{\max }\right\}$. Each transition in the NLTC trellis corresponds to $\left(B_{\max }+1\right)^{2}$ transitions in the extended trellis, however, some of these transitions might be invalid depending on the current and the next battery states, and the output label in the NLTC.

Let us illustrate this idea by a simple example. Consider a two-state NLTC given in Fig. 7. Assuming that the energy harvester is equipped with a unit-sized battery, a section of the extended trellis diagram for this code is shown in Fig. 8. The state $S_{i} / x$ corresponds to the case where the NLTC state is $S_{i}$ and the battery state is $x$. The labels corresponding to each transition of the extended trellis along with their probabilities are given in Table I where $\bar{q}=1-q$. In this approach, the BCJR decoder operates on the extended trellis and exchanges

$$
\begin{aligned}
& Y_{i} \sim\left(p_{i} \bar{\pi}_{i 0} p_{j} \bar{\pi}_{j 0}\right) \mathcal{N}\left(h_{i i}+h_{j i}, \sigma_{i}^{2}\right)+\left(p_{i} \bar{\pi}_{i 0}\left(1-p_{j} \bar{\pi}_{j 0}\right)\right) \mathcal{N}\left(h_{i i}, \sigma_{i}^{2}\right) \\
&+\left(\left(1-p_{i} \bar{\pi}_{i 0}\right) p_{j} \bar{\pi}_{j 0}\right) \mathcal{N}\left(h_{j i}, \sigma_{i}^{2}\right)+\left(\left(1-p_{i} \bar{\pi}_{i 0}\right)\left(1-p_{j} \bar{\pi}_{j 0}\right)\right) \mathcal{N}\left(0, \sigma_{i}^{2}\right) \\
& Y_{i} \mid X_{i} \sim p_{i}\left[\left(\bar{\pi}_{i 0} p_{j} \bar{\pi}_{j 0}\right) \mathcal{N}\left(h_{i i}+h_{j i}, \sigma_{i}^{2}\right)+\left(\bar{\pi}_{i 0}\left(1-p_{j} \bar{\pi}_{j 0}\right)\right) \mathcal{N}\left(h_{i i}, \sigma_{i}^{2}\right)+\left(\pi_{i 0} p_{j} \bar{\pi}_{j 0}\right) \mathcal{N}\left(h_{j i}, \sigma_{i}^{2}\right)\right. \\
&\left.\quad+\left(\pi_{i 0}\left(1-p_{j} \bar{\pi}_{j 0}\right)\right) \mathcal{N}\left(0, \sigma_{i}^{2}\right)\right]+\bar{p}_{i}\left[\left(p_{j} \bar{\pi}_{j 0}\right) \mathcal{N}\left(h_{j i}, \sigma_{i}^{2}\right)+\left(1-p_{j} \bar{\pi}_{j 0}\right) \mathcal{N}\left(0, \sigma_{i}^{2}\right)\right]
\end{aligned}
$$




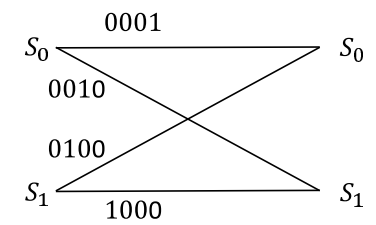

Fig. 7. The 2-state NLTC.

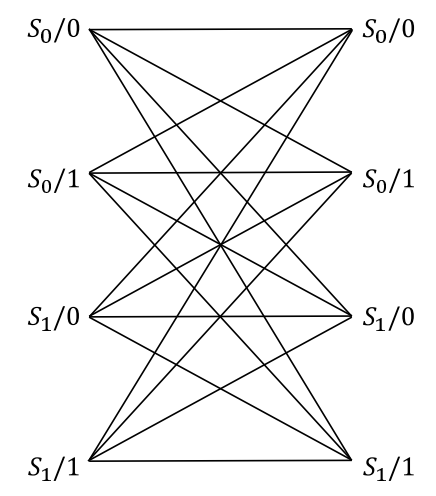

Fig. 8. The extended trellis section for 2-state NLTC and unit-sized battery.

extrinsic LLRs with the LDPC decoder in an iterative manner. Complexity increase for the improved decoder compared to the simple decoding scheme is at most quadratic which can be tolerated even for code design purposes, however, we do not employ this scheme for designing codes in this paper. We demonstrate the superior performance of the proposed solution via finite block length bit error rate simulations using specific codes taken from the designed ensembles of the previous subsection. We also note that, reduced complexity trellis-based decoders can also be employed to obtain computationally simpler decoding solutions while still utilizing the extended trellis to achieve a performance versus complexity trade-off.

\section{Extensions to the Interference Channel Set-Up}

We now consider two-user interference channels, and similar to the single-user case, we propose a practical coding scheme based on serially concatenated NLTCs with outer LDPC codes for each user. To perform the code design based on the proposed scheme, we ignore the memory in the battery states, i.e., assume that they are i.i.d. for both users. We consider two different decoding methods at the receiver side, namely single-user decoding (SUD) and joint decoding (JD). If there is interference between users, using public messages and performing joint decoding at the receivers helps with decoding of the desired messages, and enables higher rates, however, one may also use private messages and employ single-user decoding as a simpler approach.

Block diagram of the proposed coding scheme with SUD is shown in Fig. 9. The transmitter side consists of concatenation of an outer LDPC encoder with an inner NLTC. As in the single-user case, the goal of the inner NLTC is to induce the desired nonuniform distribution at the channel input while providing protection against channel noise, and the outer LDPC
TABLE I

LABELS CORRESPONDING TO THE TRANSITIONS OF THE EXTENDED TRELLIS AND THEIR PROBABILITIES

\begin{tabular}{|c|c|c|c|}
\hline Current State & Next State & Output Label & Probability \\
\hline \hline \multirow{2}{*}{$S_{0} / 0$} & \multirow{2}{*}{$S_{0} / 0$} & 0000 & $\bar{q}^{4}$ \\
\cline { 3 - 4 } & \multirow{2}{*}{$S_{0} / 0$} & 0001 & $\left(1-\bar{q}^{3}\right) \bar{q}$ \\
\hline & $S_{0} / 1$ & 0000 & $\bar{q}^{3} q$ \\
\hline$S_{0} / 1$ & $S_{0} / 0$ & 0001 & $\left(1-\bar{q}^{3}\right) q$ \\
\hline$S_{0} / 1$ & $S_{0} / 1$ & 0001 & $\bar{q}$ \\
\hline \multirow{2}{*}{$S_{0} / 0$} & \multirow{2}{*}{$S_{1} / 0$} & 0000 & $q$ \\
\cline { 3 - 4 } & & 0010 & $\left(1-\bar{q}^{2}\right) \bar{q}^{2}$ \\
\hline \multirow{2}{*}{$S_{0} / 0$} & $S_{1} / 1$ & 0000 & $\bar{q}^{2}\left(1-\bar{q}^{2}\right)$ \\
\hline & & 0010 & $\left(1-\bar{q}^{2}\right)^{2}$ \\
\hline$S_{0} / 1$ & $S_{1} / 0$ & 0010 & $\bar{q}^{2}$ \\
\hline$S_{0} / 1$ & $S_{1} / 1$ & 0010 & $1-\bar{q}^{2}$ \\
\hline$S_{1} / 0$ & \multirow{2}{*}{$S_{0} / 0$} & 0000 & $\bar{q}^{4}$ \\
\cline { 3 - 4 } & & 0100 & $q \bar{q}^{3}$ \\
\hline \multirow{2}{*}{$S_{1} / 0$} & $S_{0} / 1$ & 0000 & $\bar{q}\left(1-\bar{q}^{3}\right)$ \\
\hline$S_{1} / 1$ & $S_{0} / 0$ & 0100 & $q\left(1-\bar{q}^{3}\right)$ \\
\hline$S_{1} / 1$ & $S_{0} / 1$ & 0100 & $\bar{q}^{3}$ \\
\hline$S_{1} / 0$ & $S_{1} / 0$ & 0100 & $1-\bar{q}^{3}$ \\
\hline$S_{1} / 0$ & $S_{1} / 1$ & 0000 & $\bar{q}^{4}$ \\
\hline$S_{1} / 1$ & $S_{1} / 0$ & 1000 & $1-\bar{q}^{4}$ \\
\hline$S_{1} / 1$ & $S_{1} / 1$ & 1000 & $1-\bar{q}^{4}$ \\
\hline
\end{tabular}

code is intended to provide error correction capabilities. Each receiver decodes its intended signal while treating interference as noise. However, since the interference from the other user (which is treated as noise) is not i.i.d. (because of the trellis based code), we utilize an interleaver and de-interleaver just before and right after the channel for one of the users.

Fig. 10 depicts the block diagram of the proposed coding scheme with joint decoding at the receivers. The difference with the scheme in Fig. 9 is that we perform JD of both intended and interfering signals at the receiver side which increases the achieved rates. The idea of improved iterative decoding by including the battery state in the trellis can be implemented on the product trellis for the case of joint decoding as well, however, the required number of states increases dramatically, hence we do not implement this idea for the joint decoder, i.e., we only use the improved decoding approach for the single-user decoding algorithm.

\section{NumERICAL EXAMPLES}

\section{A. Single-User EH Communications}

As a first example, we consider a single-user energy harvesting transmitter with a unit-sized battery and energy arrival probability of $q=0.4$ communicating over an AWGN channel. We consider a ones' density of $p=0.25$ which is the optimal ones' density for this energy arrival probability with NIID strategies at high SNRs, and design an NLTC satisfying this constraint while maximizing the minimum distance of the code. Details of the designed trellis code are given in Table II. In the next step, by fixing the inner NLTC, the outer LDPC 


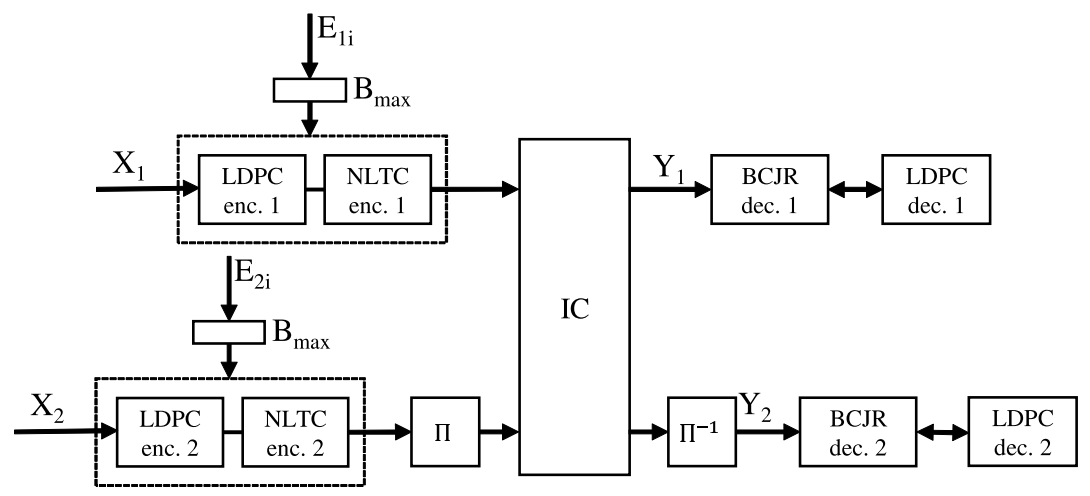

Fig. 9. Block diagram of the proposed coding scheme with SUD.

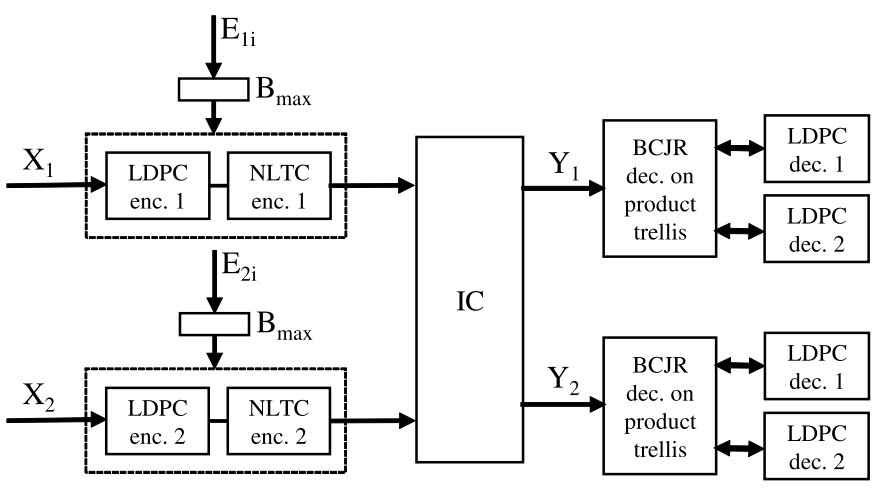

Fig. 10. Block diagram of the proposed coding scheme with JD.

TABLE II

Label Assignment to the Branches of 16-State Trellis FOR THE DESIGNED NLTC $(M=4)$

\begin{tabular}{|c|c|c|c|c|c|c|c||c|}
\hline \multicolumn{7}{|c|}{ State/Input } & Output \\
\hline \hline $0000 / 0$ & $0001 / 1$ & $0100 / 1$ & $0101 / 0$ & $1010 / 0$ & $1011 / 1$ & $1110 / 1$ & $1111 / 0$ & 0001 \\
\hline $0000 / 1$ & $0001 / 0$ & $0100 / 0$ & $0101 / 1$ & $1010 / 1$ & $1011 / 0$ & $1110 / 0$ & $1111 / 1$ & 0010 \\
\hline $0010 / 0$ & $0011 / 1$ & $0110 / 1$ & $0111 / 0$ & $1000 / 0$ & $1001 / 1$ & $1100 / 1$ & $1101 / 0$ & 0100 \\
\hline $0010 / 1$ & $0011 / 0$ & $0110 / 0$ & $0111 / 1$ & $1000 / 1$ & $1001 / 0$ & $1100 / 0$ & $1101 / 1$ & 1000 \\
\hline
\end{tabular}

code is designed by maximizing the rate of the code for a convergence threshold of $0 \mathrm{~dB}$ for which the result is given in Table III. Note also that concatenation of a nonlinear memoryless mapper (NLMM) (of the same rate and ones' density as the designed NLTC) with an optimized outer LDPC code is considered as reference.

To study the performance of the specific codes from the designed ensemble, parity check matrices for a block-length of $10 \mathrm{k}$ are obtained using the tools in [29] where the length-4 cycles are removed for improved performance. The resulting bit error rates are depicted in Fig. 11. The overall code rate for both schemes are 0.126 . We observe that the designed codes via the proposed approach outperform the reference scheme by about $2.5 \mathrm{~dB}$ at a bit error rate of $10^{-3}$. Furthermore the superior performance of the improved decoding scheme compared to the simple approach (by about $0.25 \mathrm{~dB}$ at the same bit error rate value) can be observed from the simulations.

As a second example, we consider an energy harvester with a unit-sized battery and an energy arrival probability of $q=0.34$ over a BSC with crossover probability $\epsilon=0.1$. The
TABLE III

OPTIMIZED DEgREE DisTRIBUTION OF THE OUTER LDPC CODE FOR THE PROPOSEd CODING SCHEME FOR EXAMPLES 1 AND 2

\begin{tabular}{|c||c|c|c|c|c|c|}
\hline & $\lambda_{2}$ & $\lambda_{3}$ & $\lambda_{4}$ & $\lambda_{5}$ & & \\
\hline Ex.1 (AWGN) & 0.492561 & 0.106999 & 0.399260 & 0.001180 & & \\
\hline Ex.2 (BSC) & 0.568342 & 0.067598 & 0.351750 & 0.012310 & & \\
\hline & $\rho_{2}$ & $\rho_{3}$ & $\rho_{4}$ & $\rho_{8}$ & $\rho_{14}$ & $\rho_{15}$ \\
\hline Ex.1 (AWGN) & 0.012700 & 0.405182 & 0.034768 & 0.027342 & 0.025157 & 0.494851 \\
\hline Ex.2 (BSC) & 0.000208 & 0.088826 & 0.004891 & 0.067288 & 0.254800 & 0.583986 \\
\hline
\end{tabular}

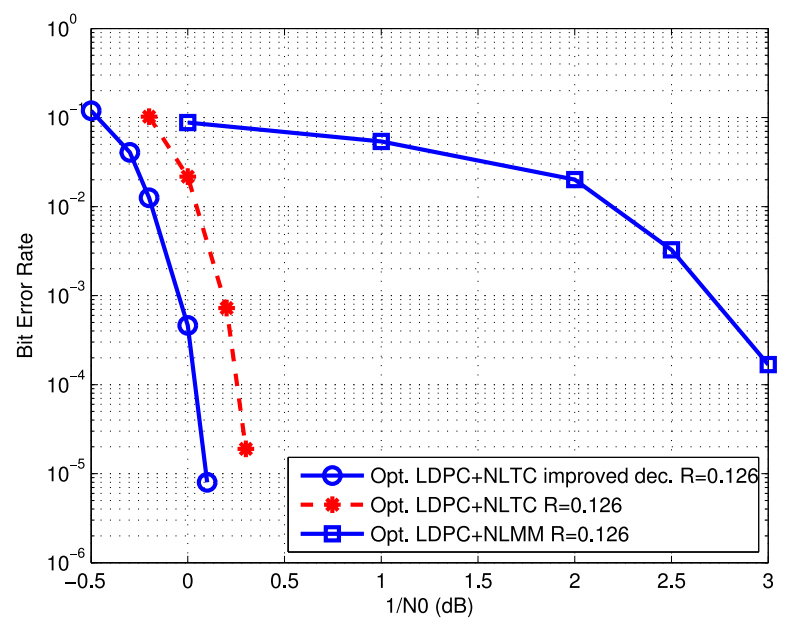

Fig. 11. Bit error rate performance of the proposed concatenated coding scheme with simple and improved decoding compared to that of the reference scheme $(q=0.4)$.

optimal ones' density for this setup is $p=0.25$, hence we use the same NLTC as in the previous example. We optimize the outer LDPC code for this channel by fixing the inner NLTC and maximizing the rate of the LDPC code ensemble (see Table III for the resulting degree distributions). The achieved rates by using the proposed and reference schemes are 0.1892 and 0.1741 , respectively. We note that the achieved rate of 0.1892 bits/channel use has a gap of only 0.009 with the achievable information theoretic limit of the NIID strategies.

We also construct specific LDPC codes with block lengths of $10 \mathrm{k}$ using the optimized degree distributions employing the tools in [29], and perform bit error rate simulations. The results are illustrated in Fig. 12. It can be observed that the newly designed codes provide clear transmission rate and error probability advantages compared to the reference scheme of using 


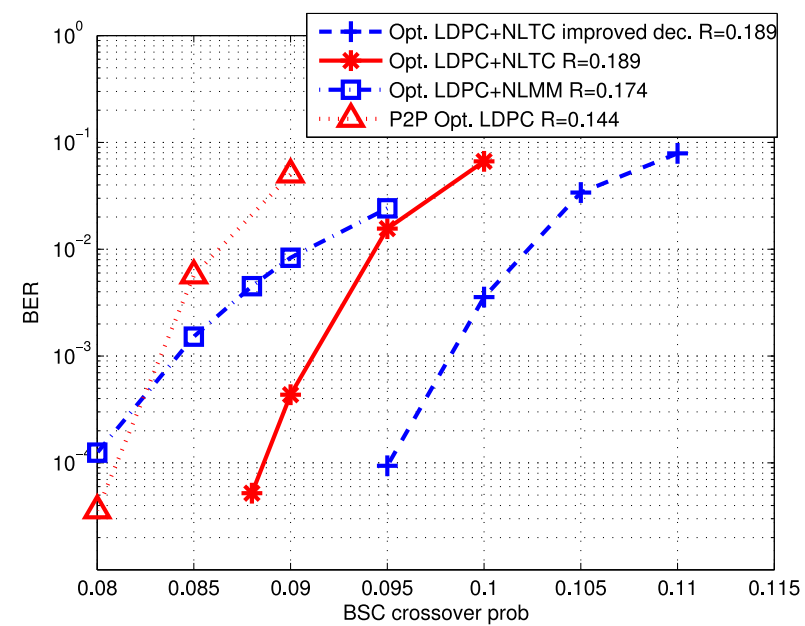

Fig. 12. Bit error rate performance of the proposed concatenated coding scheme and reference scheme $(q=0.34)$.

nonlinear memoryless mappers with optimized outer LDPC codes, and P2P optimal codes. In addition, the simulation results show that, for example, at a crossover probability of $\epsilon=0.095$, an improvement of about two orders of magnitude in the bit error rate can be obtained by using the improved iterative decoding solution incorporating the battery states compared to the simple approach which ignores them. Note that since the capacity is unknown for these examples, we do not provide a comparison with the ultimate information theoretic limits.

\section{B. Two-User EH Interference Channel}

We now turn our attention to the case of EH transmitters over an interference channel. Specifically, we consider the two-user discrete memoryless interference channel in (5) with noise parameters $\epsilon_{1}=0.21$ and $\epsilon_{2}=0.25$, unit-sized battery transmitters and energy arrival probabilities $q=0.5$. The achievable rate subregion considering i.i.d. inputs and singleuser decoding at both users is shown in Fig. 13. Note that in order to achieve a rate pair on the optimal boundary of the rate subregion, specific nonuniform distributions for both users are required. For example, considering i.i.d. inputs with a ones' density of $p=0.25$ for both users achieves the rate pair marked on the figure. The achieved rate pair is very close to the optimal boundary of the achievable rate subregion.

We now design an NLTC of memory 4 satisfying the ones' density of $p=0.25$ while maximizing the minimum distance of the code. Parameters of the designed trellis code are the same as those given in Table II. Considering the specifically designed NLTC (instead of i.i.d. nonuniform inputs) and utilizing the numerical methods in [30], we calculate the achievable rate pair with single-user decoding for the current example which is also illustrated in Fig. 13. The figure clearly illustrates the advantage of using a nonlinear trellis-based encoding instead of nonuniform i.i.d. channel inputs.

We optimize the degree distribution of the outer LDPC codes for this specific channel and the inner NLTC based on maximizing the rate of the code. The resulting degree distributions are given in Table IV. The explicitly designed codes

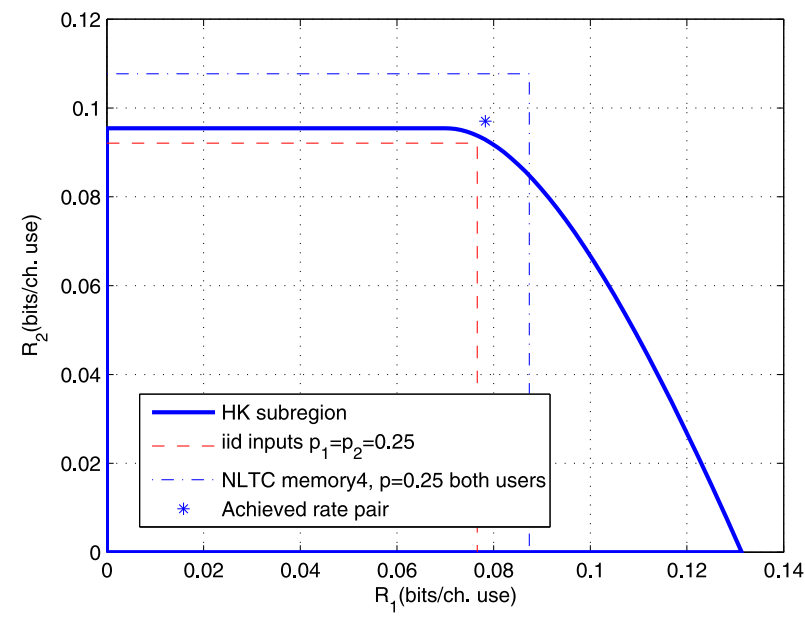

Fig. 13. Achievable rate subregion and the achieved rate pair using the proposed coding scheme with single-user decoding for two-user EHIC of (5).

TABLE IV

Optimized DEGREe Distribution OF THE OUTER LDPC CODES FOR TWO-USER EHIC (5)

\begin{tabular}{|c||c|c|c|c|c|c|}
\hline & $\lambda_{2}$ & $\lambda_{3}$ & $\lambda_{4}$ & $\lambda_{5}$ & & \\
\hline User 1 & 0.251016 & 0.235664 & 0.046370 & 0.466950 & & \\
\hline User 2 & 0.219598 & 0.286582 & 0.025020 & 0.468800 & & \\
\hline & $\rho_{2}$ & $\rho_{3}$ & $\rho_{4}$ & $\rho_{8}$ & $\rho_{14}$ & $\rho_{15}$ \\
\hline User 1 & 0.134175 & 0.271975 & 0.029495 & 0.144806 & 0.220355 & 0.199195 \\
\hline User 2 & 0.073385 & 0.299035 & 0.001771 & 0.121574 & 0.247337 & 0.256898 \\
\hline
\end{tabular}

achieve a rate pair of $(0.07831,0.09705)$ which is marked on the figure. We notice that this rate pair cannot be achieved without using nonlinear codes, which clearly illustrates the importance of using NLTC codes.

To study the performance of the specific codes from the designed ensemble, parity check matrices for a block-length of 10k are obtained using the tools in [29] where the length-4 cycles are removed for improved performance. Parity check matrices for P2P optimal LDPC codes with same rate as the overall rate of the concatenated coding scheme are also obtained. The selected codes are simulated over a twouser EHIC (5) for which the resulting bit error rates are depicted in Fig. 14. The BER simulation results show that the sample codes exhibit a waterfall behavior close to the estimated thresholds, and they significantly outperform the P2P alternatives. In particular, for the first user, which is prone to interference, the performance gain is more significant. Furthermore, the superior performance of the improved decoding scheme compared to the simple one (by about an order of magnitude for the same crossover probability) can be observed.

As another example, we consider the same two-user EHIC in (5) with slightly different noise parameters $\epsilon_{1}=0.15$ and $\epsilon_{2}=0.25$, unit-sized battery transmitters and energy arrival probabilities $q=0.5$ (referred as Example 4). We utilize both SUD and JD at the first user's receiver.

The ARRs corresponding to the proposed scheme are depicted in Fig. 15, which clearly show that using a public message for the second user and performing joint decoding at the first user's receiver improves the achievable rate subregion 

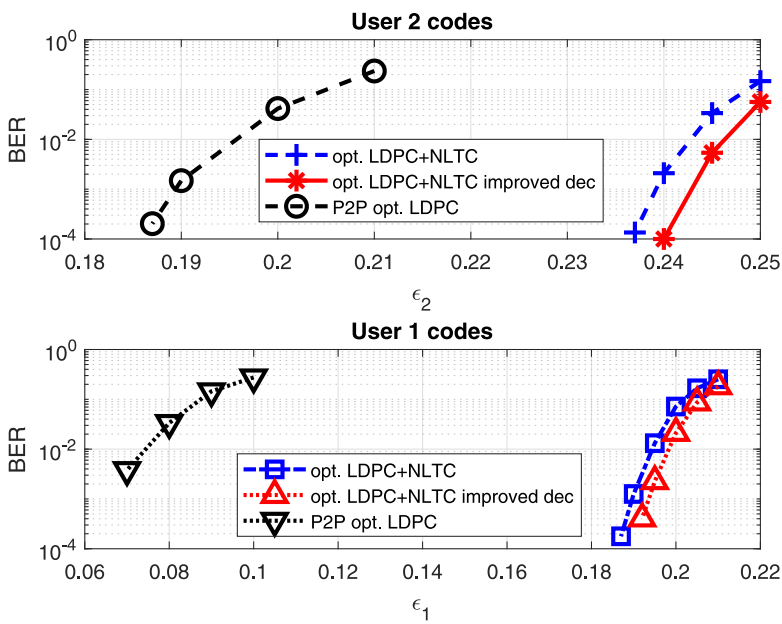

Fig. 14. Bit error rate performance of the designed codes with simple and improved decoding approaches and that of the P2P optimal LDPC codes.

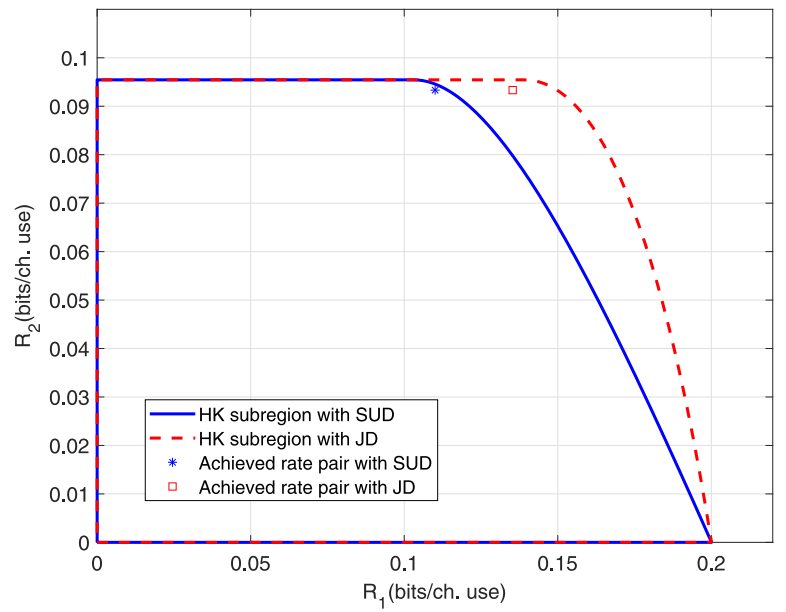

Fig. 15. Achievable rate subregion and the achieved rate pair using the proposed coding scheme with single-user decoding and joint decoding for Example 4.

TABLE V

LABel AsSignment to the BRAnChes of 4-State Trellis $(M=2)$ FOR THE DESIGNED NLTC

\begin{tabular}{|c|c||c|}
\hline \multicolumn{2}{|c||}{ State/Input } & Output \\
\hline \hline $00 / 0$ & $01 / 1$ & 0001 \\
\hline $00 / 1$ & $01 / 0$ & 0010 \\
\hline $10 / 0$ & $11 / 1$ & 0100 \\
\hline $10 / 1$ & $11 / 0$ & 1000 \\
\hline
\end{tabular}

TABLE VI

Optimized DegReE Distribution OF THE OUter LDPC Codes FOR EXAMPLE 4

\begin{tabular}{|c||c|c|c|c|c|c|c|}
\hline & $\lambda_{2}$ & $\lambda_{3}$ & $\lambda_{4}$ & $\lambda_{5}$ & & & \\
\hline User 1(SUD) & 0.206124 & 0.296626 & 0.030490 & 0.466760 & & & \\
\hline User 1(JD) & 0.247980 & 0.533610 & 0.139760 & 0.078650 & & & \\
\hline User 2 & 0.237414 & 0.111726 & 0.038380 & 0.612480 & & & \\
\hline & $\rho_{2}$ & $\rho_{3}$ & $\rho_{4}$ & $\rho_{5}$ & $\rho_{8}$ & $\rho_{14}$ & $\rho_{15}$ \\
\hline User 1(SUD) & 0.000848 & 0.305336 & 0.064133 & & 0.140212 & 0.243930 & 0.245541 \\
\hline User 1(JD) & 0.005507 & 0.138682 & 0.048959 & 0.281425 & 0.155395 & 0.027638 & 0.342395 \\
\hline User 2 & 0.010801 & 0.296790 & 0.118995 & & 0.118142 & 0.276270 & 0.179002 \\
\hline
\end{tabular}

significantly. For specific code design, we select a ones' density of $p=0.25$ for both users, however, we consider using an NLTC of memory 2 in order to reduce the complexity of

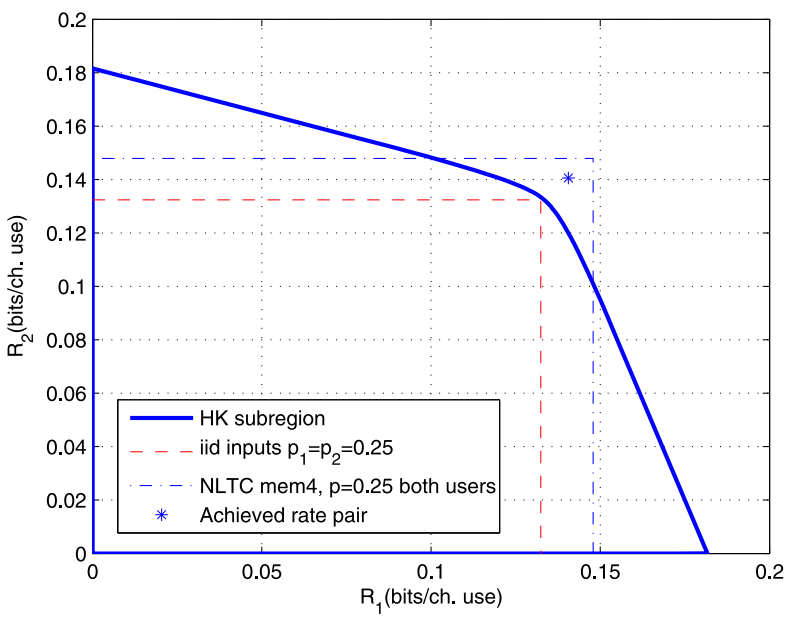

Fig. 16. Achievable rate subregion and the achieved rate pair using the proposed coding scheme for Example 5.

TABLE VII

Optimized DEGREe Distribution OF THE OUTER LDPC CODES FOR EXAMPLE 5

\begin{tabular}{|l||c|c|c|c|c|c|}
\hline & $\lambda_{2}$ & $\lambda_{3}$ & $\lambda_{4}$ & $\lambda_{5}$ & & \\
\hline User 1 and 2 & 0.610905 & 0.007875 & 0.001550 & 0.379670 & & \\
\hline & $\rho_{2}$ & $\rho_{3}$ & $\rho_{4}$ & $\rho_{8}$ & $\rho_{14}$ & $\rho_{15}$ \\
\hline User 1 and 2 & 0.002293 & 0.302971 & 0.079697 & 0.071308 & 0.216830 & 0.326903 \\
\hline
\end{tabular}

the joint decoder. Table $\mathrm{V}$ shows the details of the designed NLTC. We optimize the outer LDPC codes for both decoding schemes, resulting in the rate pairs of $(0.1101,0.0933)$ and $(0.1353,0.09333)$ for SUD and JD, respectively (see Table VI for the resulting degree distributions). The achieved rate pairs are shown in Fig. 15, which demonstrates that by using the proposed approach with joint decoding we can achieve rate pairs that cannot be achieved by single-user decoding alone.

As a final example, we consider a two-user EH Gaussian interference channel with $q=0.5, S N R_{1}=1, S N R_{2}=1$, $I N R_{1}=0.5$ and $I N R_{2}=0.5$ (Example 5). We obtain the achievable rate subregion for this channel utilizing SUD with i.i.d. inputs as depicted in Fig. 16. The achievable rate pairs with i.i.d inputs for a ones' density of $p=0.25$, and the NLTC in Table II are also shown. The results demonstrate that utilizing the NLTC instead of i.i.d. inputs results in higher achievable transmission rates. Furthermore, by designing the outer LDPC code for the specific NLTC, we achieve a rate pair of $R_{1}=R_{2}=0.1406$ bits/channel use (also marked on the same figure). Degree distribution of the designed code is given in Table VII. Note that since the channel is symmetric, the same code works for both users.

\section{CONCLUSION}

We have considered a two-user EH communication system with interference. For both cases of DMICs and GICs, we have obtained achievable rate regions based on HK coding with i.i.d. on-off signaling for both single-user decoding and joint decoding approaches. We observed that increasing the battery size enlarges the ARR, indeed, even by using a unit battery at the transmitters, we can achieve a significantly large portion of the ARR corresponding to no EH constraints. We have also 
proposed a practical coding solution utilizing a serially concatenated coding scheme with an inner NLTC and outer LDPC code. Furthermore, we have developed a decoding method utilizing the memory in the system, and we have shown via extensive numerical examples that employing the designed codes, we can achieve rate pairs close to the information theoretic achievable rate region boundaries. The results also demonstrate that the newly designed codes significantly outperform the alternatives of using P2P optimal codes and the reference scheme of utilizing nonlinear memoryless mappers with specifically optimized LDPC codes.

\section{REFERENCES}

[1] O. Ozel and S. Ulukus, "Achieving AWGN capacity under stochastic energy harvesting," IEEE Trans. Inf. Theory, vol. 58, no. 10, pp. 6471-6483, Oct. 2012.

[2] O. Ozel and S. Ulukus, "AWGN channel under time-varying amplitude constraints with causal information at the transmitter," in Proc. Conf. Rec. 45th Asilomar Conf. Signals Syst. Comput. (ASILOMAR), Pacific Grove, CA, USA, Nov. 2011, pp. 373-377.

[3] W. Mao and B. Hassibi, "Capacity analysis of discrete energy harvesting channels," IEEE Trans. Inf. Theory, vol. 63, no. 9, pp. 5850-5885, Sep. 2017.

[4] D. Shaviv, P.-M. Nguyen, and A. Özgür, "Capacity of the energyharvesting channel with a finite battery," IEEE Trans. Inf. Theory, vol. 62, no. 11, pp. 6436-6458, Nov. 2016.

[5] V. Jog and V. Anantharam, "An energy harvesting AWGN channel with a finite battery," in Proc. IEEE Int. Symp. Inf. Theory (ISIT), Honolulu, HI, USA, Jun. 2014, pp. 806-810.

[6] K. Tutuncuoglu, O. Ozel, A. Yener, and S. Ulukus, "The binary energy harvesting channel with a unit-sized battery," IEEE Trans. Inf. Theory, vol. 63, no. 7, pp. 4240-4256, Jul. 2017.

[7] T. M. Duman and M. Stojanovic, "Information rates of energy harvesting communications with intersymbol interference," IEEE Commun. Lett., vol. 23, no. 12, pp. 2164-2167, Dec. 2019.

[8] J. Yang and S. Ulukus, "Optimal packet scheduling in an energy harvesting communication system," IEEE Trans. Commun., vol. 60, no. 1, pp. 220-230, Jan. 2012.

[9] K. Tutuncuoglu and A. Yener, "Optimum transmission policies for battery limited energy harvesting nodes," IEEE Trans. Wireless Commun., vol. 11, no. 3, pp. 1180-1189, Mar. 2012.

[10] O. Orhan, D. Gündüz, and E. Erkip, "Throughput maximization for an energy harvesting communication system with processing cost," in Proc. IEEE Inf. Theory Workshop (ITW), Lausanne, Switzerland, Sep. 2012, pp. 84-88.

[11] P. Blasco, D. Gündüz, and M. Dohler, "A Learning theoretic approach to energy harvesting communication system optimization," IEEE Trans. Wireless Commun., vol. 12, no. 4, pp. 1872-1882, Apr. 2013.

[12] H. A. Inan, D. Shaviv, and A. Özgür, "Capacity of the energy harvesting Gaussian MAC," IEEE Trans. Inf. Theory, vol. 64, no. 4, pp. 2347-2360, Apr. 2018.

[13] A. Baknina and S. Ulukus, "Energy harvesting multiple access channels: Optimal and near-optimal online policies," IEEE Trans. Commun., vol. 66, no. 7, pp. 2904-2917, Jul. 2018

[14] M. K. Sharma, C. R. Murthy, and R. Vaze, "Asymptotically optimal uncoordinated power control policies for energy harvesting multiple access channels with decoding costs," IEEE Trans. Commun., vol. 67, no. 3, pp. 2420-2435, Mar. 2019.

[15] K. Tutuncuoglu and A. Yener, "Sum-rate optimal power policies for energy harvesting transmitters in an interference channel," J. Commun. Netw., vol. 14, no. 2, pp. 151-161, Apr. 2012.

[16] D. K. Shin, W. Choi, and D. I. Kim, "The two-user Gaussian interference channel with energy harvesting transmitters: Energy cooperation and achievable rate region," IEEE Trans. Commun., vol. 63, no. 11, pp. 4551-4564, Nov. 2015.

[17] M. Dabirnia and T. M. Duman, "Code design for binary energy harvesting channel," in Proc. IEEE Int. Symp. Inf. Theory (ISIT), Jun. 2017, pp. 1127-1131.

[18] M. Dabirnia, "Coding schemes for energy harvesting and multi-user communications," Ph.D. dissertation, Dept. Elect. Electron. Eng., Bilkent Univ., Ankara, Turkey, Dec. 2017.
[19] M. Ozates and T. M. Duman, "Channel coding for energy harvesting communications using run length limited codes," in Proc. IEEE Glob. Commun. Conf. (GLOBECOM), Abu Dhabi, UAE, Dec. 2018, pp. 1-6.

[20] G. Böcherer and R. Mathar, "Operating LDPC codes with zero shaping gap," in Proc. IEEE Inf. Theory Workshop, Paraty, Brazil, 2011, pp. 330-334.

[21] A. M. Fouladgar, O. Simeone, and E. Erkip, "Constrained codes for joint energy and information transfer," IEEE Trans. Commun., vol. 62, no. 6, pp. 2121-2131, Jun. 2014

[22] C. E. Shannon, "Channels with side information at the transmitter," $I B M$ J. Rese. Dev., vol. 2, no. 4, pp. 289-293, 1958.

[23] M. Dabirnia and T. M. Duman, "On the code design for joint energy and information transfer," IEEE Trans. Commun., vol. 64, no. 6, pp. 2677-2688, Jun. 2016.

[24] M. Dabirnia, A. K. Tanc, S. Sharifi, and T. M. Duman, "Code design for discrete memoryless interference channel," IEEE Trans. Commun., vol. 66, no. 8, pp. 3368-3380, Aug. 2018.

[25] S. T. Brink, "Convergence behavior of iteratively decoded parallel concatenated codes," IEEE Trans. Commun., vol. 49, no. 10, pp. 1727-1737, Oct. 2001.

[26] J. Hagenauer, "The exit chart-introduction to extrinsic information transfer in iterative processing," in Proc. 12th Eur. Signal Process. Conf., Vienna, Austria, Sep. 2004, pp. 1541-1548.

[27] J. Hou, P. Siegel, L. Milstein, and H. Pfister, "Capacity-approaching bandwidth-efficient coded modulation schemes based on low-density parity-check codes," IEEE Trans. Inf. Theory, vol. 49, no. 9, pp. 2141-2155, Sep. 2003.

[28] S. Sharifi, A. K. Tanc, and T. M. Duman, "Implementing the Han-Kobayashi scheme using low density parity check codes over Gaussian interference channels," IEEE Trans. Commun., vol. 63, no. 2, pp. 337-350, Feb. 2015.

[29] IT++. [Online]. Available: http://itpp.sourceforge.net/4.3.1/

[30] D. M. Arnold, H. A. Loeliger, P. O. Vontobel, A. Kavcic, and W. Zeng, "Simulation-based computation of information rates for channels with memory," IEEE Trans. Inf. Theory, vol. 52, no. 8, pp. 3498-3508, Aug. 2006.

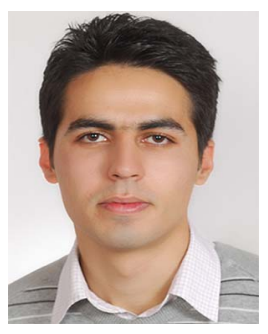

Mehdi Dabirnia (Member, IEEE) received the B.S. and M.S. degrees in electrical engineering from the University of Tehran, Tehran, Iran, in 2007 and 2010, respectively, and the Ph.D. degree in electrical engineering from Bilkent University, Ankara, Turkey, in 2017. He is currently a Postdoctoral Researcher with the Department of Information and Communication Technologies, Universitat Pompeu Fabra, Barcelona, Spain. His research interests include various topics in communication theory, information theory, and coding theory.

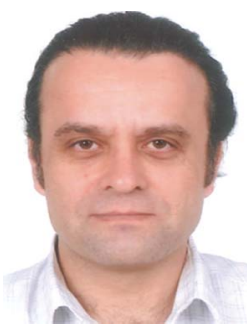

Tolga M. Duman (Fellow, IEEE) received the B.S. degree in electrical engineering from Bilkent University, Ankara, Turkey, in 1993, and the M.S. and $\mathrm{Ph} . \mathrm{D}$. degrees in electrical engineering from Northeastern University, Boston, MA, USA, in 1995 and 1998, respectively. He was with the School of ECEE, Arizona State University as an Assistant Professor from 1998 to 2004, an Associate Professor from 2004 to 2008, and a Professor after 2008. He is a Professor with the Electrical and Electronics Engineering Department, Bilkent University, Turkey. His current research interests are in systems, with particular focus on communication and signal processing, including wireless and mobile communications, coding/modulation, coding for wireless communications, data storage systems, and underwater acoustic communications. He is a recipient of the National Science Foundation CAREER Award and the IEEE Third Millennium medal. He is currently the Editor-in-Chief of the IEEE TRANSACTIONS ON COMMUNICATIONS. 